\title{
The use of two relations in $L$-fuzzy Contexts
}

\author{
C.Alcalde ${ }^{\mathrm{a}, *}$, A.Burusco $^{\mathrm{b}}$, R.Fuentes-González ${ }^{\mathrm{b}}$ \\ ${ }^{a}$ Escuela Universitaria Politécnica. Departamento de Matemática Aplicada. Universidad \\ del País Vasco. UPV/EHU. Plaza de Europa 1, 20018 San Sebastián, Spain. \\ ${ }^{b}$ Departamento de Automática y Computación. Universidad Pública de Navarra, Campus \\ de Arrosadía, 31006 Pamplona, Spain.
}

\begin{abstract}
The main goal of the paper is the study of the $L$-fuzzy Concept Analysis when the $L$-fuzzy context $(L, X, Y, R, Q)$ uses two $L$-fuzzy relations to represent the relationship between the objects $X$ and the attributes $Y$. This generalization will be called $L$-fuzzy bicontext. This situation is very usual in different disciplines of social sciences.

We study the $L$-fuzzy concepts of these $L$-fuzzy bicontexts and we obtain some interesting results. Specifically, we will be able to classify the biconcepts of the $L$-fuzzy bicontext.

Finally, a practical case is developed using this new tool.

Keywords: Formal concept analysis, $L$-fuzzy concept analysis, knowledge acquisition
\end{abstract}

\section{Introduction}

This paper presents a generalization of the $L$-fuzzy Concept Analysis when the $L$-fuzzy context $(L, X, Y, R, Q)$ uses two relations $R \in L^{X \times Y}$ and $Q \in L^{Y \times X}$ to set up the relation between the set of objects $X$ and the set of attributes $Y$.

This situation is very common in social sciences, to analyze the human behavior, or in philosophy. Also in economy, to make studies of preference relations (when $X=Y$ ).

\footnotetext{
*Corresponding author. Tel:+34 943017226, fax:+34 943017130.

Email addresses: c.alcalde@ehu.es (C.Alcalde), burusco@unavarra.es (A.Burusco), rfuentes@unavarra.es (R.Fuentes-González)
} 
We will see that the process of obtaining the $L$-fuzzy concepts is more complex in this situation than in the usual $L$-fuzzy concept analysis but we will use the results of $\mathrm{P}$. and $\mathrm{R}$. Cousot to present new algorithms of calculation.

We also propose a new method that allows to classify the $L$-fuzzy concepts of this new $L$-fuzzy context and to establish similarity relations between the objects and the attributes.

The idea of defining an $L$-fuzzy context from other two given contexts has previously been treated in other papers such as [14], where the authors defined the $L$-bonds and the direct product of two $L$-fuzzy contexts, or in [1] where the composition of $L$-fuzzy contexts was analyzed. However, the problem presented in this work is noticeably different because the relationship between two elements will depend on the point of view of the considered element.

We will complete the section with the main results about this subject.

\subsection{The Formal Concept Analysis}

R. Wille in his Formal Concept Analysis [21] proposes a new theory based on the three basic ideas of the conceptual knowledge: the objects, the attributes and the concepts. These ideas are joined through three basic relations: one object has an attribute, one object belongs to a concept and one concept is a subconcept of another one. With this analysis a new model to represent the concepts and to set up hierarchies among them is defined.

The Formal Concept Analysis of R. Wille [21, 12] extracts information from a binary table that represents a formal context $(X, Y, R)$ where $X$ and $Y$ are two finite sets of objects and attributes respectively and $R \subseteq X \times Y$ is a binary relation defined between them. The hidden information is obtained by means of the formal concepts which are pairs $(A, B)$ with $A \subseteq X, B \subseteq Y$ verifying $A^{\star}=B$ and $B^{\star}=A$, where $\star$ is the derivation operator that associates the attributes related to the elements of $A$ to every object set $A$, and the objects related to the attributes of $B$ to every attribute set $B$. These formal concepts can be interpreted as a group of objects $A$ that shares the attributes of $B$. 


\subsection{L-fuzzy concept analysis}

In previous papers $[7,9]$ we have defined the $L$-fuzzy contexts $(L, X, Y, R)$, with $L$ a complete lattice, $X$ and $Y$ sets of objects and attributes respectively and $R \in L^{X \times Y}$ an $L$-fuzzy relation between the objects and the attributes. This is an extension of the Wille's formal contexts to the fuzzy case when the relationship between the objects and the attributes takes values in a complete lattice $L$, instead of being binary values.

In our case, the new L-fuzzy concepts $(A, B) \in L^{X} \times L^{Y}$ are pairs obtained as follows:

First, we defined the derivation operators $(\cdot)_{1}$ and $(\cdot)_{2}$ given by means of the following expressions, to work with these $L$-fuzzy contexts:

For any $A \in L^{X}$ and $B \in L^{Y}$,

$$
\begin{aligned}
& A_{1}(y)=\inf _{x \in X}\{I(A(x), R(x, y))\} \\
& B_{2}(x)=\inf _{y \in Y}\{I(B(y), R(x, y))\}
\end{aligned}
$$

with $I$ a fuzzy implication operator defined in the lattice $(L, \leq)$ and where $A_{1}$ represents the attributes related to the objects of $A$ in a fuzzy way, and $B_{2}$ represents the objects related to all the attributes of $B$.

The defined $L$-fuzzy subsets $A_{1}$ and $B_{2}$ are said to be the fuzzy extension and the fuzzy intension respectively, and can be expressed by means of the triangle operator $\triangleleft[13]$ associated with the implication operator $I$ as:

$$
A_{1}=A \triangleleft R, \quad B_{2}=B \triangleleft R^{o p},
$$

where $R^{o p} \in L^{Y \times X}$ is the opposite relation of $R$.

The information stored in the context is visualized by means of the $L$ fuzzy concepts that are pairs $\left(A, A_{1}\right) \in L^{X} \times L^{Y}$ where $A$ is a fixed point of the operator $\varphi$, which is defined from the derivation operators $(\cdot)_{1}$ and $(\cdot)_{2}$ as $\varphi(A)=\left(A_{1}\right)_{2}=A_{12}$. These pairs, whose first and second components are said to be the fuzzy extension and intension respectively, represent a set of objects that share a set of attributes in a fuzzy way.

The set $\mathcal{L}=\left\{\left(A, A_{1}\right) / A \in \operatorname{fix}(\varphi)\right\}$, where fix $(\varphi)$ represents the set of fixed points of the operator $\varphi$, with the order relation $\leq$ defined as:

$$
\left(A, A_{1}\right) \leq\left(C, C_{1}\right) \text { if } A \leq C\left(\text { or } A_{1} \geq C_{1}\right)
$$

is a complete lattice that is said to be the $L$-fuzzy concept lattice $[7,9]$. 
On the other hand, considering $A \in L^{X}$, (or $B \in L^{Y}$ ) we can obtain the associated $L$-fuzzy concept. In the case of using a residuated implication, the associated $L$-fuzzy concept is $\left(A_{12}, A_{1}\right)$ (or $\left(B_{2}, B_{21}\right)$ ).

Other important papers that generalize the Formal Concepts Analysis using residuated implication operators are due to $\mathrm{R}$. Belohlavek $[3,4,5]$ and S. Pollandt [18]. Moreover, extensions of the Formal Concept Analysis to the interval-valued case are in $[2,11]$ and to the fuzzy property-oriented and multi-adjoint concept lattices framework in $[16,15,17]$.

The rest of the paper is organized as follows: Section 2 tackles a general study of the $L$-fuzzy bicontexts and the definition of the $L$-fuzzy biconcept. Section 3 studies the construction of the $L$-fuzzy biconcept lattice. In Section 4 the problem of the classification of the $L$-fuzzy biconcepts is analyzed. Finally, the conclusions and future work are detailed in Section 5. Furthermore, interesting examples can be found throughout the paper.

\section{2. $\quad L$-fuzzy Bicontexts}

Let $(L, \leq)$ be a complete lattice with cardinality $|L| \geq 2$. Consider $X$ and $Y$ two non empty sets.

Definition 1. The tuple $(L, X, Y, R, Q)$ with $R \in L^{X \times Y}$ and $Q \in L^{Y \times X}$, is said to be an L-fuzzy bicontext where $X$ and $Y$ are the object and attribute sets respectively, and $R$ and $Q$ represent the relationship between the elements of $X$ and $Y$.

Let us see an example to introduce these new contexts:

Example 1. Consider the $L$-fuzzy bicontext $(L, X, Y, R, Q)$ where $X$ and $Y$ are two groups of people. The lattice is $L=\{0,0.5,1\}$, and the relations $R \in L^{X \times Y}$ and $Q \in L^{Y \times X}$ represent the opinion of the people of $X$ about $Y$ and the people of $Y$ about $X$ respectively (see Table 1 ). The value 1 means "like" and the value 0 means "don't like" or "dislike".

In order to study the $L$-fuzzy concepts of these new $L$-fuzzy bicontexts, we will define the derivation operator associated with an $L$-fuzzy relation as follows: 
Table 1: Opinion of the people of each of the groups about the other one

\begin{tabular}{c|ccccc}
$R$ & $y_{1}$ & $y_{2}$ & $y_{3}$ & $y_{4}$ & $y_{5}$ \\
\hline$x_{1}$ & 1 & 0 & 0.5 & 0.5 & 0 \\
$x_{2}$ & 0 & 1 & 1 & 0 & 0 \\
$x_{3}$ & 0.5 & 0 & 0 & 1 & 1 \\
$x_{4}$ & 1 & 0 & 0 & 0.5 & 1
\end{tabular}

\begin{tabular}{c|cccc}
$Q$ & $x_{1}$ & $x_{2}$ & $x_{3}$ & $x_{4}$ \\
\hline$y_{1}$ & 1 & 0 & 0.5 & 1 \\
$y_{2}$ & 0 & 1 & 0 & 0 \\
$y_{3}$ & 0 & 1 & 1 & 0 \\
$y_{4}$ & 1 & 0 & 0.5 & 1 \\
$y_{5}$ & 0 & 0 & 0 & 1
\end{tabular}

Definition 2. Let $X_{1}$ and $X_{2}$ be two finite sets and let us consider $S \in$ $L^{X_{1} \times X_{2}}$ an $L$-fuzzy relation between them. The derivation operator associated with the relation $S,(\cdot)_{S}$, is defined as:

$$
\text { For every } A \in L^{X_{1}} \text { and } z \in X_{2}, \quad A_{S}(z)=\inf _{t \in X_{1}}\{I(A(t), S(t, z))\}
$$

where $I: L \times L \longrightarrow L$ is an $L$-fuzzy implication operator.

The L-fuzzy set $A_{S}$ represents, in a fuzzy way, the set of elements of $X_{2}$ related by $S$ to all the elements of $A$.

Hence, in the $L$-fuzzy bicontext $(L, X, Y, R, Q)$, we can consider two derivation operators which expressions are:

For any $A \in L^{X}$ and $B \in L^{Y}$,

$$
\begin{aligned}
& A_{R}(y)=\inf _{x \in X}\{I(A(x), R(x, y))\} \\
& B_{Q}(x)=\inf _{y \in Y}\{I(B(y), Q(y, x))\}
\end{aligned}
$$

with $I: L \times L \longrightarrow L$ an $L$-fuzzy implication operator.

$A_{R}$ represents the set of attributes related to all the objects of $A$ in $R$, in a fuzzy way, and $B_{Q}$, the objects related to all the attributes of $B$ in $Q$.

\section{Remark 1.}

a) If $Q=R^{o p}$ then the $L$-fuzzy bicontext $(L, X, Y, R, Q)$ and the $L$-fuzzy context $(L, X, Y, R)$ are coincident. In this case, the derivation operators defined on the $L$-fuzzy context are such that $(\cdot)_{1}=(\cdot)_{R}$ and $(\cdot)_{2}=(\cdot)_{R^{o p}}$. 
b) If $R$ is a symmetrical relation and $Q=R$, then the $L$-fuzzy bicontext $(L, X, Y, R, Q)$ and the $L$-fuzzy context $(L, X, Y, R)$ are coincident.

¿From this point forward, in order to simplify the notation, we are going to denote the iterative application of derivation operators $\left((\cdot)_{S}\right)_{T \ldots}$ by $(\cdot)_{S T \ldots}$.

These derivation operators verify the following properties.

\section{Proposition 1.}

a) The derivation operators $(\cdot)_{R}$ and $(\cdot)_{Q}$ are decreasing:

For every $A, C \in L^{X}$ and $B, D \in L^{Y}$,

$$
\begin{aligned}
& A \leq C \Longrightarrow A_{R} \geq C_{R} \\
& B \leq D \Longrightarrow B_{Q} \geq D_{Q}
\end{aligned}
$$

b) Consider $A \in L^{X}$. In general, $A \not \leq A_{R Q}$ and $A_{R Q R} \neq A_{R}$.

In the same way, if $B \in L^{Y}$, in general, $B \not \leq B_{Q R}$ and $B_{Q R Q} \neq B_{Q}$.

c) For any family of $L$-fuzzy subsets $\left\{A_{j} \in L^{X} \mid j \in J\right\}$ it is verified that

$$
\left(\bigvee_{j \in J} A_{j}\right)_{R} \leq \bigwedge_{j \in J}\left(A_{j}\right)_{R}
$$

Analogously, for any $\left\{B_{k} \in L^{Y} \mid k \in K\right\}$,

$$
\left(\bigvee_{k \in K} B_{k}\right)_{Q} \leq \bigwedge_{k \in K}\left(B_{k}\right)_{Q}
$$

d) If the implication I verifies that for any $M \subseteq L$ and $\beta \in L$,

$$
I\left(\bigvee_{\alpha \in M} \alpha, \beta\right)=\bigwedge_{\alpha \in M} I(\alpha, \beta)
$$

then,

$$
\begin{aligned}
& \left(\bigvee_{j \in J} A_{j}\right)_{R}=\bigwedge_{j \in J}\left(A_{j}\right)_{R} \\
& \left(\bigvee_{k \in K} B_{k}\right)_{Q}=\bigwedge_{k \in K}\left(B_{k}\right)_{Q}
\end{aligned}
$$




\section{PROOF.}

a) It is obvious taking into account that the implication operator is decreasing in the first argument.

b) We can go back to Example 1 and take the following counterexample: Let $A=\left\{x_{1} / 1, x_{2} / 0, x_{3} / 0, x_{4} / 0\right\}$ be an $L$-fuzzy subset of $X$. Then, using the Lukasiewicz implication operator $I$ for the definition of the derivation operator,

$$
\begin{aligned}
& A_{R}=\left\{y_{1} / 1, y_{2} / 0, y_{3} / 0.5, y_{4} / 0.5, y_{5} / 0\right\} \\
& A_{R Q}=\left\{x_{1} / 0.5, x_{2} / 0, x_{3} / 0.5, x_{4} / 0.5\right\} \\
& A_{R Q R}=\left\{y_{1} / 1, y_{2} / 0.5, y_{3} / 0.5, y_{4} / 1, y_{5} / 0.5\right\}
\end{aligned}
$$

Hence, $A \not \leq A_{R Q}$ and $A_{R Q R} \neq A_{R}$.

We can also find a counterexample taking as a starting point $B=$ $\left\{y_{1} / 0, y_{2} / 0, y_{3} / 1, y_{4} / 0, y_{5} / 0\right\} \in L^{Y}$.

c) For every $j_{0} \in J$, it is verified that $A_{j_{0}} \geq \bigwedge_{j \in J} A_{j}$.

Applying the derivation operator: $\left(A_{j_{0}}\right)_{R} \leq\left(\bigwedge_{j \in J} A_{j}\right)_{R}$, then $\left(\bigwedge_{j \in J} A_{j}\right)_{R}$ is an upper bound of the set $\left\{\left(A_{j}\right)_{R}, j \in J\right\}$. As the supremum is the least upper bound, then $\bigvee_{j \in J}\left(A_{j}\right)_{R} \leq\left(\bigwedge_{j \in J} A_{j}\right)_{R}$.

The other inequality is proved in the same way.

d) For any $y \in Y,\left(\bigvee_{j \in J} A_{j}\right)_{R}(y)=\inf _{x \in X}\left\{I\left(\bigvee_{j \in J} A_{j}(x), R(x, y)\right)\right\}$

Applying the hypothesis

$\inf _{x \in X}\left\{I\left(\bigvee_{j \in J} A_{j}(x), R(x, y)\right)\right\}=\inf _{x \in X}\left\{\bigwedge_{j \in J}\left(I\left(A_{j}(x), R(x, y)\right)\right)\right\}$

and, as $L$ is a complete lattice,

$\inf _{x \in X}\left\{\bigwedge_{j \in J}\left(I\left(A_{j}(x), R(x, y)\right)\right)\right\}=\bigwedge_{j \in J}\left\{\inf _{x \in X}\left(I\left(A_{j}(x), R(x, y)\right)\right)\right\}=\bigwedge_{j \in J}\left(A_{j}\right)_{R}$

The proof for $B_{k} \in L^{Y}$ is similar to this one. 
Corollary 1. The maps $\varphi: L^{X} \longrightarrow L^{X}$ and $\psi: L^{Y} \longrightarrow L^{Y}$ defined for any $A \in L^{X}$ and $B \in L^{Y}$ as:

$$
\begin{aligned}
& \varphi(A)=A_{R Q} \\
& \psi(B)=B_{Q R}
\end{aligned}
$$

are increasing.

Therefore, by the Theorem of Tarski [19], the fixed point sets:

$$
\begin{aligned}
& \operatorname{fix}(\varphi)=\left\{A \in L^{X} / \varphi(A)=A\right\} \\
& \operatorname{fix}(\psi)=\left\{B \in L^{Y} / \psi(B)=B\right\}
\end{aligned}
$$

endowed with the usual orders in $L^{X}$ and $L^{Y}$ are complete lattices, which will be denoted by $\Omega=\left(\operatorname{fix}(\varphi), \leq, 0_{\Omega}, 1_{\Omega}, \vee_{\Omega}, \wedge_{\Omega}\right)$ and $\Sigma=\left(\operatorname{fix}(\psi), \geq, 0_{\Sigma}, 1_{\Sigma}, \vee_{\Sigma}, \wedge_{\Sigma}\right)$.

Definition 3. The operators $\varphi$ and $\psi$ defined above are called constructor operators.

Remark 2. In general, by Proposition 1.b), the constructor operators $\varphi$ and $\psi$ are not closure operators.

Using the derivation operator, we introduce the following definition.

Definition 4. The pair $(A, B) \in L^{X} \times L^{Y}$ such that $A_{R}=B$ and $B_{Q}=A$ is said to be an $L$-fuzzy biconcept of the $L$-fuzzy bicontext $(L, X, Y, R, Q)$.

Example 2. In Example 1 the pair:

$$
\left(\left\{x_{1} / 0.5, x_{2} / 0, x_{3} / 0.5, x_{4} / 1\right\},\left\{y_{1} / 1, y_{2} / 0, y_{3} / 0, y_{4} / 0.5, y_{5} / 0.5\right\}\right)
$$

is an $L$-fuzzy biconcept.

In this case, we can interpret the meaning of this concept saying that $x_{4}$ and $y_{1}$ like each other. And with a lower exigence level, we can also say that everybody in $\left\{x_{1}, x_{3}, x_{4}\right\}$ likes $y_{1}, y_{4}$ and $y_{5}$, and vice versa.

The next property establishes the relationship between these $L$-fuzzy biconcepts and the fixed point sets of $\varphi$ and $\psi$.

Proposition 2. The following statements are equivalent:

a) The pair $(A, B) \in L^{X} \times L^{Y}$ is an L-fuzzy biconcept of the L-fuzzy bicontext $(L, X, Y, R, Q)$. 
b) $\varphi(A)=A$ and $B=A_{R}$.

c) $\psi(B)=B$ and $A=B_{Q}$.

Proof. It is an immediate consequence of the definition of $L$-fuzzy biconcept.

Remark 3. In $L$-fuzzy Concept Analysis, when the used implication operator $I$ is residuated, the obtained constructor operators are closure operators. However, this property is not fulfilled in the case of working with $L$-fuzzy biconcepts. As a consequence, the process of obtaining the fixed point sets starting from $L$-fuzzy sets $A$ needs the application of derivation operators a non fixed number of times.

In general, it is not determined how many times is needed to derive in order to find the fixed point. For instance, in the next example we can see that the use of operators $(\cdot)_{R Q}$ is needed five times to find this fixed point.

Example 3. Consider the $L$-fuzzy bicontext $(L, X, Y, R, Q)$ where the lattice $L=\{0,0.1,0.2, \ldots, 1\}$, and the relations $R \in L^{X \times Y}, Q \in L^{Y \times X}$ are represented in Table 2 .

Table 2: Relations of the $L$-fuzzy bicontex of Example 3

\begin{tabular}{|c|c|c|c|c|c|c|c|}
\hline$R$ & $y_{1}$ & $y_{2}$ & $y_{3}$ & $Q$ & $x_{1}$ & $x_{2}$ & $x_{3}$ \\
\hline$x_{1}$ & 0.3 & 0.9 & 0.2 & $y_{1}$ & 0.8 & 0.3 & 0.9 \\
\hline$x_{2}$ & 1 & 0.5 & 0.2 & $y_{2}$ & 0.2 & 0 & 1 \\
\hline$x_{3}$ & 0.4 & 0.8 & 1 & $y_{3}$ & 0.8 & 0 & 0.3 \\
\hline
\end{tabular}

Let $A=\left\{x_{1} / 0.8, x_{2} / 1, x_{3} / 0.5\right\}$ be an $L$-fuzzy subset of $X$. Then, using the Lukasiewicz implication operator $I$ for the definition of the derivation operator,

$$
\begin{aligned}
& A_{R Q}=\left\{x_{1} / 0.7, x_{2} / 0.5, x_{3} / 1\right\} \\
& A_{R Q R Q}=\left\{x_{1} / 0.4, x_{2} / 0.2, x_{3} / 0.8\right\} \\
& A_{R Q R Q R Q}=\left\{x_{1} / 0.2, x_{2} / 0, x_{3} / 0.5\right\} \\
& A_{R Q R Q R Q R Q}=\left\{x_{1} / 0.2, x_{2} / 0, x_{3} / 0.3\right\} \\
& A_{R Q R Q R Q R Q R Q}=\left\{x_{1} / 0.2, x_{2} / 0, x_{3} / 0.3\right\}
\end{aligned}
$$


Therefore, $A_{R Q R Q R Q R Q R Q}=A_{R Q R Q R Q R Q}$.

\section{The $L$-fuzzy biconcept lattice}

We will begin this section with the analysis of the existing hierarchy among the $L$-fuzzy biconcepts of the $L$-fuzzy bicontext.

Theorem 1. Let $\mathcal{L}(L, X, Y, R, Q)$ be the L-fuzzy biconcept set endowed with the order relation $\preceq$ defined as:

$$
(A, B) \preceq(C, D) \Longleftrightarrow A \leq C(\text { or } B \geq D)
$$

Then, $(\mathcal{L}(L, X, Y, R, Q), \preceq)$ is a complete lattice.

Proof. Consider $\mathcal{F}=\left\{\left(A_{j},\left(A_{j}\right)_{R}\right) \mid A_{j} \in \operatorname{fix}(\varphi), j \in J\right\} \subseteq \mathcal{L}(L, X, Y, R, Q)$ a family of $L$-fuzzy biconcepts. Let us prove that the supremum of $\mathcal{F}$ exists.

As $\Omega=(\operatorname{fix}(\varphi), \leq)$ is a complete lattice, then $\bigvee_{j \in J} A_{j} \in \operatorname{fix}(\varphi)$ and hence, for any $j \in J,\left(A_{j},\left(A_{j}\right)_{R}\right) \preceq\left(\bigvee_{j \in J} A_{j},\left(\bigvee_{j \in J} A_{j}\right)_{R}\right)$.

Moreover $\left(\bigvee_{\Omega} A_{j},\left(\bigvee_{\Omega} A_{j}\right)_{R}\right)$ is the least upper bound:

If $\left(M, M_{R}\right)$ is another upper bound then, for any $j \in J, M \geq A_{j}$. So, $M \geq \bigvee_{j \in J} A_{j}$ and therefore, $\left(\bigvee_{\Omega} A_{j},\left(\bigvee_{\Omega} A_{j}\right)_{R}\right) \preceq\left(M, M_{R}\right)$.

Analogously, it can be proved that the $L$-fuzzy biconcept $\left(\bigwedge_{j \in J} A_{j},\left(\bigwedge_{j \in J} A_{j}\right)_{R}\right)$ is the infimum of $\mathcal{F}$.

This theorem can be proved in a similar way using the fixed points of the operator $\psi$. Thus, if the family $\mathcal{F}$ is represented as $\mathcal{F}=\left\{\left(\left(B_{j}\right)_{Q}, B_{j}\right) \mid B_{j} \in\right.$ $\operatorname{fix}(\psi), j \in J\}$, the obtained expressions for the supremum and the infimum elements of $\mathcal{F}$ are:

$$
\begin{aligned}
& \bigvee_{\mathcal{L}} \mathcal{F}=\left(\left(\bigwedge_{\Sigma} B_{j}\right)_{Q}, \bigwedge_{\Sigma} B_{j}\right) \\
& \bigwedge_{\mathcal{L}} \mathcal{F}=\left(\left(\bigvee_{\Sigma} B_{j}\right)_{Q}, \bigvee_{\Sigma} B_{j}\right)
\end{aligned}
$$

Definition 5. The lattice $(\mathcal{L}(L, X, Y, R, Q), \preceq)$ is said to be the $L$-fuzzy biconcept lattice of the L-fuzzy bicontext $(L, X, Y, R, Q)$. 
Proposition 3. The minimum and maximum elements of the lattice of $L$ fuzzy biconcepts $(\mathcal{L}(L, X, Y, R, Q), \preceq)$ are $0_{\mathcal{L}}=\left(0_{\Omega}, 1_{\Sigma}\right)$ and $1_{\mathcal{L}}=\left(1_{\Omega}, 0_{\Sigma}\right)$.

Proof. Consider $A \in \operatorname{fix}(\varphi)$. Then $0_{\Omega} \leq A \leq 1_{\Omega}$. Applying the derivation operator, $\left(0_{\Omega}\right)_{R} \geq A_{R} \geq\left(1_{\Omega}\right)_{R}$.

As $\operatorname{fix}(\psi)=\left\{A_{R} / A \in \operatorname{fix}(\varphi)\right\}$, we can conclude that $\left(0_{\Omega}\right)_{R}=1_{\Sigma}$ and $\left(1_{\Omega}\right)_{R}=0_{\Sigma}$.

Then, the minimum element in $\mathcal{L}$ has the expression $0_{\mathcal{L}}=\left(0_{\Omega},\left(0_{\Omega}\right)_{R}\right)=$ $\left(0_{\Omega}, 1_{\Sigma}\right)$ and the maximum $1_{\mathcal{L}}=\left(1_{\Omega},\left(1_{\Omega}\right)_{R}\right)=\left(1_{\Omega}, 0_{\Sigma}\right)$.

Example 4. Returning to Example 1, the obtained $L$-fuzzy biconcept lattice is formed by the following $L$-fuzzy biconcepts:

$$
\begin{aligned}
& \left(A_{1}, B_{1}\right)=\left(\left\{x_{1} / 0, x_{2} / 0, x_{3} / 0, x_{4} / 0\right\},\left\{y_{1} / 1, y_{2} / 1, y_{3} / 1, y_{4} / 1, y_{5} / 1\right\}\right) \\
& \left(A_{2}, B_{2}\right)=\left(\left\{x_{1} / 0, x_{2} / 0.5, x_{3} / 0, x_{4} / 0\right\},\left\{y_{1} / 0.5, y_{2} / 1, y_{3} / 1, y_{4} / 0.5, y_{5} / 0.5\right\}\right) \\
& \left(A_{3}, B_{3}\right)=\left(\left\{x_{1} / 0, x_{2} / 0, x_{3} / 0, x_{4} / 0.5\right\},\left\{y_{1} / 1, y_{2} / 0.5, y_{3} / 0.5, y_{4} / 1, y_{5} / 1\right\}\right) \\
& \left(A_{4}, B_{4}\right)=\left(\left\{x_{1} / 0.5, x_{2} / 0, x_{3} / 0.5, x_{4} / 0.5\right\},\left\{y_{1} / 1, y_{2} / 0.5, y_{3} / 0.5, y_{4} / 1, y_{5} / 0.5\right\}\right) \\
& \left(A_{5}, B_{5}\right)=\left(\left\{x_{1} / 0, x_{2} / 1, x_{3} / 0, x_{4} / 0\right\},\left\{y_{1} / 0, y_{2} / 1, y_{3} / 1, y_{4} / 0, y_{5} / 0\right\}\right) \\
& \left(A_{6}, B_{6}\right)=\left(\left\{x_{1} / 0.5, x_{2} / 0.5, x_{3} / 0.5, x_{4} / 0.5\right\},\left\{y_{1} / 0.5, y_{2} / 0.5, y_{3} / 0.5, y_{4} / 0.5, y_{5} / 0.5\right\}\right) \\
& \left(A_{7}, B_{7}\right)=\left(\left\{x_{1} / 0, x_{2} / 0, x_{3} / 0, x_{4} / 1\right\},\left\{y_{1} / 1, y_{2} / 0, y_{3} / 0, y_{4} / 0.5, y_{5} / 1\right\}\right) \\
& \left(A_{8}, B_{8}\right)=\left(\left\{x_{1} / 0.5, x_{2} / 0, x_{3} / 0.5, x_{4} / 1\right\},\left\{y_{1} / 1, y_{2} / 0, y_{3} / 0, y_{4} / 0.5, y_{5} / 0.5\right\}\right) \\
& \left(A_{9}, B_{9}\right)=\left(\left\{x_{1} / 0.5, x_{2} / 1, x_{3} / 0.5, x_{4} / 0.5\right\},\left\{y_{1} / 0, y_{2} / 0.5, y_{3} / 0.5, y_{4} / 0, y_{5} / 0\right\}\right) \\
& \left(A_{10}, B_{10}\right)=\left(\left\{x_{1} / 0.5, x_{2} / 0.5, x_{3} / 0.5, x_{4} / 1\right\},\left\{y_{1} / 0.5, y_{2} / 0, y_{3} / 0, y_{4} / 0.5, y_{5} / 0.5\right\}\right) \\
& \left(A_{11}, B_{11}\right)=\left(\left\{x_{1} / 1, x_{2} / 0, x_{3} / 0.5, x_{4} / 1\right\},\left\{y_{1} / 1, y_{2} / 0, y_{3} / 0, y_{4} / 0.5, y_{5} / 0\right\}\right) \\
& \left(A_{12}, B_{12}\right)=\left(\left\{x_{1} / 1, x_{2} / 0.5, x_{3} / 1, x_{4} / 1\right\},\left\{y_{1} / 0.5, y_{2} / 0, y_{3} / 0, y_{4} / 0.5, y_{5} / 0\right\}\right) \\
& \left(A_{13}, B_{13}\right)=\left(\left\{x_{1} / 1, x_{2} / 1, x_{3} / 1, x_{4} / 1\right\},\left\{y_{1} / 0, y_{2} / 0, y_{3} / 0, y_{4} / 0, y_{5} / 0\right\}\right)
\end{aligned}
$$

This lattice can be graphically represented by Figure 1 .

The supremum and the infimum of the lattice $(\mathcal{L}(L, X, Y, R, Q), \preceq)$ can also be expressed by means of the supremum and infimum in the lattices $\Omega$ and $\Sigma$ as follows:

Theorem 2. Let I be an implication operator verifying $I\left(\bigvee_{\alpha \in M} \alpha, \beta\right)=\bigwedge_{\alpha \in M} I(\alpha, \beta)$, for any $M \subseteq L$ and $\beta \in L$. 


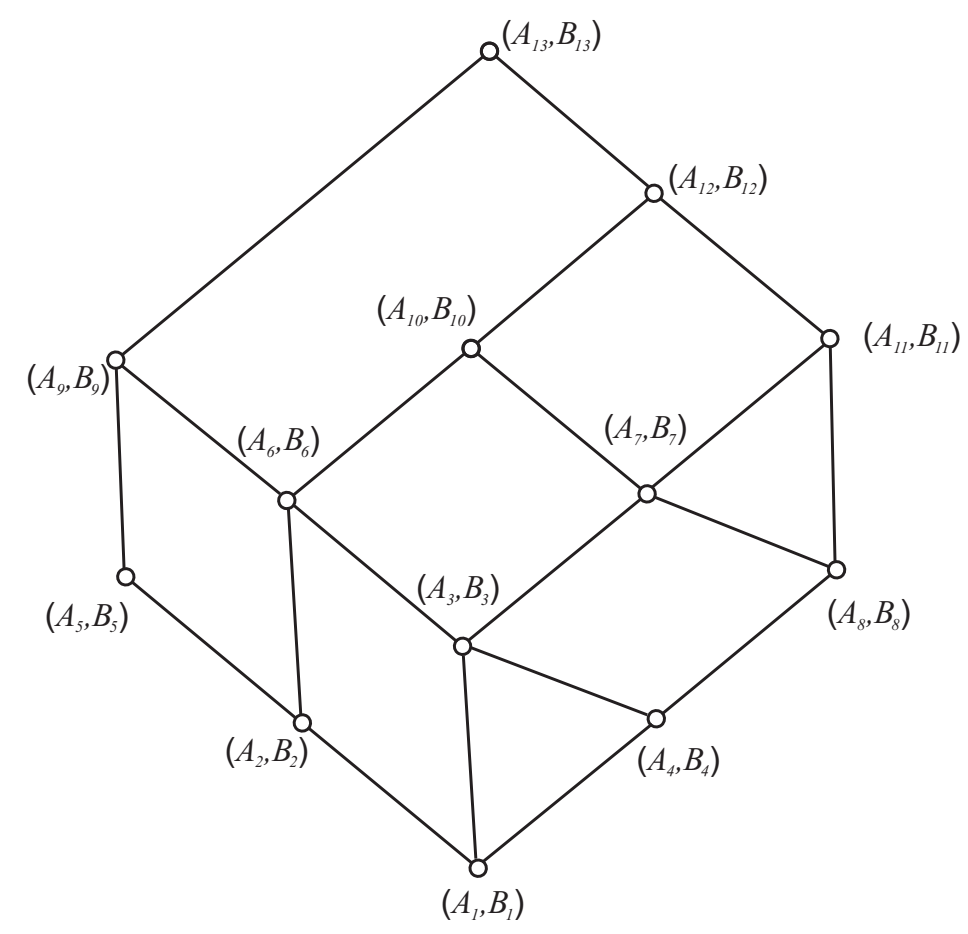

Figure 1: $L$-fuzzy biconcept lattice of Example 4.

Consider $\mathcal{F}=\left\{\left(A_{j},\left(A_{j}\right)_{R}\right), A_{j} \in \operatorname{fix}(\varphi), j \in J\right\}=\left\{\left(\left(B_{j}\right)_{Q}, B_{j}\right)\right), B_{j} \in$ $\operatorname{fix}(\psi), j \in J\} \subseteq \mathcal{L}$

The supremum and the infimum of $\mathcal{F}$ are:

$$
\begin{aligned}
& \bigvee_{\mathcal{L}}\left(A_{j},\left(A_{j}\right)_{R}\right)=\left(\bigvee_{\Omega} A_{j}, \bigwedge_{\Sigma}\left(A_{j}\right)_{R}\right) \\
& \bigwedge_{\mathcal{L}}\left(\left(B_{j}\right)_{Q}, B_{j}\right)=\left(\bigwedge_{\Omega}\left(B_{j}\right)_{Q}, \bigvee_{\Sigma} B_{j}\right)
\end{aligned}
$$

Proof. Immediate taking into account Theorem 1 and Proposition 1.

In order to obtain the $L$-fuzzy biconcepts of the lattice $\mathcal{L}(L, X, Y, R, Q)$, that is, the fixed points of the constructor operators $\varphi$ and $\psi$, we will appeal to the work developed by P. Cousot and R. Cousot [10] that provides a constructive version of Tarski's theorem which is able to calculate them by means of limits of stationary transfinite iteration sequences. 
P. Cousot and R. Cousot [10] define a transfinite iteration sequence as the one obtained by the iterative application of a monotone operator $F$ to a starting element $D$ :

$$
\left(D, F(D), F^{2}(D), F^{3}(D), \ldots\right)
$$

If $F$ is an increasing operator, the iterative sequence is said to be an upper iteration sequence, and it is a lower iteration sequence when the operator is decreasing.

The sequence $\left(X_{1}, X_{2}, \ldots\right)$ is stationary if and only if $\exists k$ such that for any $i>k, X_{i}=X_{k}$. In this case the limit of the sequence is $X_{k}$.

We denote by luis $(F)(D)$ the limit of a stationary upper iteration sequence for $F$ starting with $D$ (dually $l l i s(F)(D)$ the limit of a lower iteration sequence).

With these definitions P. Cousot and R. Cousot [10] prove some results among which we find the constructive version of Tarki's lattice theoretical fixed point theorem:

Theorem 3. The set of fixed points of $F$ is a nonempty complete lattice with ordering $\subseteq$, infimum luis $(F)(0)$, supremum llis $(F)(1)$, least upper bound luis $(F)\left(\cup S_{i}\right)$ and greatest lower bound llis $(F)\left(\cup S_{i}\right)$.

Applying this theorem to our case, we have:

$$
\begin{array}{lr}
0_{\Omega}=\operatorname{luis}(\varphi)(0) & 0_{\Sigma}=\operatorname{luis}(\psi)(0) \\
1_{\Omega}=\operatorname{llis}(\varphi)(1) & 1_{\Sigma}=\operatorname{lisis}(\psi)(1)
\end{array}
$$

Where by "0" we are representing the $L$-fuzzy subset in which all the elements have membership value 0 (that is, the subset $\emptyset$ ). The symbol " 1 " represents the $L$-fuzzy subset with all the membership values equal to 1 (the set $X$ or , respectively, $Y$ ).

In addition, for any subset of fixed points $\left\{A_{j}, j \in J\right\} \subset \operatorname{fix}(\varphi)$ and $\left\{B_{j}, j \in J\right\} \subset \operatorname{fix}(\psi)$,

$$
\begin{array}{ll}
\bigvee_{\Omega} A_{j}=\operatorname{luis}(\varphi)\left(\bigvee A_{j}\right) & \bigvee_{\Sigma} B_{j}=\operatorname{luis}(\psi)\left(\bigvee B_{j}\right) \\
\bigwedge_{\Omega} A_{j}=\operatorname{llis}(\varphi)\left(\bigwedge A_{j}\right) & \bigwedge_{\Sigma} B_{j}=\operatorname{llis}(\psi)\left(\bigwedge B_{j}\right)
\end{array}
$$

where in general, luis $(f)(A)$ with $f$ a function that preserves the order, is the limit of a stationary upper iteration sequence for $f$ starting from $A$ :

$$
\operatorname{luis}(f)(A)=\lim \sup \left(A, f(A), f^{2}(A), f^{3}(A) \ldots\right)
$$


and llis $(f)(A)$, the limit of a stationary lower iteration sequence for $f$ starting from $A$ :

$$
\operatorname{llis}(f)(A)=\liminf \left(A, f(A), f^{2}(A), f^{3}(A) \ldots\right)
$$

Example 5. In the lattice of the example, consider the elements of $\operatorname{fix}(\varphi)$ $A=\left\{x_{1} / 0.5, x_{2} / 1, x_{3} / 0.5, x_{4} / 0.5\right\}$ and $C=\left\{x_{1} / 1, x_{2} / 0.5, x_{3} / 1, x_{4} / 1\right\}$.

In this case, the supremum in the lattice $L^{X} \times L^{Y}$ is

$$
A \vee C=\left\{x_{1} / 0.5, x_{2} / 1, x_{3} / 0.5, x_{4} / 1\right\} \notin \operatorname{fix}(\varphi)
$$

However, by the previous theorem, in the lattice of the fixed points the supremum is

$$
A \bigvee_{\Omega} C=\operatorname{luis}(\varphi)(A \vee C)=\left\{x_{1} / 1, x_{2} / 1, x_{3} / 1, x_{4} / 1\right\}
$$

Other important result to obtain the $L$-fuzzy concepts starting from $A \in$ $L^{X}$ or $B \in L^{Y}$ is the following [10]:

Theorem 4. For every $A \in L^{X}$, the $L$-Fuzzy sets $\operatorname{luis}(\varphi) \circ \operatorname{llis}\left(f_{2}\right)(A)$ and llis $(\varphi)$ oluis $\left(f_{1}\right)(A)$ are fixed points of $\varphi$ verifying $\operatorname{luis}(\varphi) \circ \operatorname{llis}\left(f_{2}\right)(A) \leq \operatorname{llis}(\varphi) \circ$ luis $\left(f_{1}\right)(A)$, where

$$
f_{1}(A)=A \vee \varphi(A) \text { and } f_{2}(A)=A \wedge \varphi(A)
$$

Moreover, these fixed points are greater than or equal to any fixed point of $\varphi$ less than or equal to $A$, and less than or equal to any fixed point of $\varphi$ greater than or equal to $A$.

These fixed points are respectively denoted by $A^{\wedge}$ and $A^{\vee}$.

$A$ similar result can be obtained starting from $B \in L^{Y}$ and using the operator $\psi$.

Example 6. If we come back to Example 1 and take as a starting set $A=$ $\left\{x_{1} / 0, x_{2} / 0, x_{3} / 0, x_{4} / 1\right\}$, then by the Cousot and Cousot operators we obtain, using the Lukasiewicz implication operator, an only fixed point $A^{\wedge}=A^{\vee}=$ $\left\{x_{1} / 0, x_{2} / 0, x_{3} / 0, x_{4} / 1\right\}$ and so, an only $L$-fuzzy biconcept:

$$
\left(\left\{x_{1} / 0, x_{2} / 0, x_{3} / 0, x_{4} / 1\right\},\left\{y_{1} / 1, y_{2} / 0, y_{3} / 0, y_{4} / 0.5, y_{5} / 1\right\}\right)
$$


However, if we take $A=\left\{x_{1} / 1, x_{2} / 0, x_{3} / 0, x_{4} / 0\right\}$, then the obtained fixed points are $A^{\wedge}=\left\{x_{1} / 0, x_{2} / 0, x_{3} / 0, x_{4} / 0\right\}$ and $A^{\vee}=\left\{x_{1} / 0.5, x_{2} / 0, x_{3} / 0.5, x_{4} / 0.5\right\}$, and the $L$-fuzzy biconcepts:

$$
\begin{gathered}
\left(\left\{x_{1} / 0, x_{2} / 0, x_{3} / 0, x_{4} / 0\right\},\left\{y_{1} / 1, y_{2} / 1, y_{3} / 1, y_{4} / 1, y_{5} / 1\right\}\right) \\
\left(\left\{x_{1} / 0.5, x_{2} / 0, x_{3} / 0.5, x_{4} / 0.5\right\},\left\{y_{1} / 1, y_{2} / 0.5, y_{3} / 0.5, y_{4} / 1, y_{5} / 0.5\right\}\right)
\end{gathered}
$$

This last $L$-fuzzy biconcept is the one obtained taking the set $A$ and applying successively the derivation operators.

Remark 4. We can also extend these results to pairs of relations $(R, Q) \in$ $L^{W \times X} \times L^{W \times Y}$ in the labeled $L$-fuzzy bicontext $(L, W, X, Y, R, Q)$ [8]. In this case, the set of labels $W$ is used to represent some important aspects of the study and is related to the object and attribute sets.

At this point, it can be interesting the study of the relationship between the $L$-fuzzy biconcepts of $(L, X, Y, R, Q)$ and the $L$-fuzzy concepts of $(L, X, Y, R)$ and $(L, Y, X, Q)$. Let us start establishing the following notation:

Notation. Given the $L$-fuzzy contexts $(L, X, Y, R)$ and $(L, Y, X, Q)$, we will denote:

$\mathcal{L}_{R Q}^{\cap}=\left\{(A, B) \in L^{X} \times L^{Y} \mid(A, B) \in \mathcal{L}(L, X, Y, R)\right.$ and $\left.(B, A) \in \mathcal{L}(L, Y, X, Q)\right\}$

$\mathcal{L}_{R Q}^{\cup}=\left\{(A, B) \in L^{X} \times L^{Y} \mid(A, B) \in \mathcal{L}(L, X, Y, R)\right.$ or $\left.(B, A) \in \mathcal{L}(L, Y, X, Q)\right\}$

Theorem 5. It is verified that:

a) $\mathcal{L}_{R Q}^{\cap} \subseteq \mathcal{L}(L, X, Y, R, Q)$.

b) $\mathcal{L}(L, X, Y, R, Q)$ is not necessarily a subset of $\mathcal{L}_{R Q}^{\cup}$.

PROOF.

a) If $(A, B) \in \mathcal{L}_{R Q}^{\cap}$, then $B=A_{R}, A=B_{R^{o p}}$ and $A=B_{Q}, B=A_{Q^{o p}}$.

So, by $B=A_{R}$ and $A=B_{Q}$, we can say that $(A, B) \in \mathcal{L}(L, X, Y, R, Q)$.

b) If we return to the lattice of Example 1, we see that $\left(\left\{x_{1} / 0, x_{2} / 0, x_{3} / 0, x_{4} / 1\right\},\left\{y_{1} / 1, y_{2} / 0, y_{3} / 0, y_{4} / 0.5, y_{5} / 1\right\}\right) \in \mathcal{L}(L, X, Y, R, Q)$. However we can prove that it does not belong to $\mathcal{L}(L, X, Y, R)$ neither to $\mathcal{L}(L, Y, X, Q)$. 
Moreover, we can set up a relation between the $L$-fuzzy concepts using $R$ and $Q$ and using the opposite $R^{o p}$ and $Q^{o p}$. It is obvious that if $R$ and $Q$ are symmetrical relations, then for every pair $(A, B) \in L^{X} \times L^{Y}$,

$$
(A, B) \in \mathcal{L}(L, X, Y, R, Q) \Longleftrightarrow(B, A) \in \mathcal{L}\left(L, Y, X, R^{o p}, Q^{o p}\right)
$$

In general, the following proposition is verified.

\section{Proposition 4.}

a) If $(A, B) \in \mathcal{L}_{R Q}^{\cap} \subseteq \mathcal{L}(L, X, Y, R, Q)$ then $(B, A) \in \mathcal{L}_{R^{o p} Q^{o p}}^{\cap} \subseteq \mathcal{L}\left(L, Y, X, R^{o p}, Q^{o p}\right)$.

b) There exists $(C, D) \in \mathcal{L}(L, X, Y, R, Q)$ such that $(D, C) \notin \mathcal{L}\left(L, Y, X, R^{o p}, Q^{o p}\right)$.

\section{PROOF.}

a) If $(A, B) \in \mathcal{L}_{R Q}^{\cap}$ we have to prove that $(B, A) \in \mathcal{L}_{R^{o p} Q^{o p}}^{\cap}$

Since $B_{R^{o p}}=\left(A_{R}\right)_{R^{o p}}=A_{R R^{o p}}=A$ and $A_{R^{o p}}=\left(B_{R}\right)_{R^{o p}}=B_{R R^{o p}}=B$, hence $(B, A) \in \mathcal{L}\left(L, Y, X, R^{o p}\right)$. In the same way, we can prove that $(B, A) \in \mathcal{L}\left(L, X, Y, Q^{o p}\right)$.

b) For instance, returning to Example 1 we can consider the element $(C, D) \in \mathcal{L}(L, X, Y, R, Q)$ such that:

$(C, D)=\left(\left\{x_{1} / 0.5, x_{2} / 0.5, x_{3} / 0.5, x_{4} / 1\right\},\left\{y_{1} / 0.5, y_{2} / 0, y_{3} / 0, y_{4} / 0.5, y_{5} / 0.5\right\}\right)$

In this case, $(D, C) \notin \mathcal{L}\left(L, Y, X, R^{o p}, Q^{o p}\right)$.

\section{Classification of the $L$-fuzzy biconcepts}

In many occasions, the large cardinality of the $L$-fuzzy biconcept lattice hinders the analysis of the contained information. In these situations, as in the case of working in an $L$-fuzzy concept lattice, it is very important to find a method to organize the information: we could classify the $L$-fuzzy biconcepts taking into account the membership degree of the objects (a similar process might be done from the point of view of the attributes).

We will try to adapt to this case the idea we proposed in [6] for $L$-fuzzy concept lattices.

Let us begin by defining a new relation $\equiv$ : 
Definition 6. Consider the L-fuzzy biconcept lattice $\mathcal{L}$.

For every $(A, B),(C, D) \in \mathcal{L}$, we can define the relation $\equiv$ as:

$$
(A, B) \equiv(C, D) \Longleftrightarrow A^{-1}\left\{\left(\min _{x \in X} A(x), 1\right]\right\}=C^{-1}\left\{\left(\min _{x \in X} C(x), 1\right]\right\}
$$

where $A^{-1}\{(\alpha, 1]\}=\{x \in X / A(x)>\alpha\}, \alpha \in L$.

Proposition $\mathbf{5}$. $\equiv$ is an equivalence relation.

ProOF. It is obvious taking into account the definition of relation $\equiv$.

Then, we can establish a partition in $\mathcal{L}$ such that $\mathcal{L}=\bigcup_{j \in J} \mathcal{C}_{j}$.

Associated with each class $\mathcal{C}_{j}=\left\{\left(A_{j, h}, B_{j, h}\right), h \in\left\{1, \ldots, k_{j}\right\}\right\}$, and fixed $h_{0} \in\left\{1, \ldots, k_{j}\right\}$, we can obtain the set $E_{j}=A_{j, h_{0}}^{-1}\left\{\left(\min _{x \in X} A_{j, h_{0}}(x), 1\right]\right\}$ to represent this class. Observe that, due to the equivalence between the elements of $\mathcal{C}_{j}$, the obtained set $E_{j}$ is the same independently of the chosen $h_{0}$.

These sets $E_{j}$ are the sets of objects that stand out from the others in class $\mathcal{C}_{j}$ looking at the membership degrees.

Example 7. The relation given in Definition 6 establish a classification for the $L$-fuzzy biconcept lattice represented in Figure 1 in order to organize its $L$-fuzzy biconcepts.

If we apply this equivalence relation $\equiv$ to the $L$-fuzzy bicontext defined in Example 1, the obtained different classes are (see Figure 2):

- $\operatorname{Class} \mathcal{C}_{1}: E_{1}=\emptyset$

$$
\begin{aligned}
& \left(A_{1,1}, B_{1,1}\right)=\left(\left\{x_{1} / 0, x_{2} / 0, x_{3} / 0, x_{4} / 0\right\},\left\{y_{1} / 1, y_{2} / 1, y_{3} / 1, y_{4} / 1, y_{5} / 1\right\}\right) \\
& \left(A_{1,2}, B_{1,2}\right)=\left(\left\{x_{1} / 0.5, x_{2} / 0.5, x_{3} / 0.5, x_{4} / 0.5\right\},\left\{y_{1} / 0.5, y_{2} / 0.5, y_{3} / 0.5, y_{4} / 0.5, y_{5} / 0.5\right\}\right) \\
& \left(A_{1,3}, B_{1,3}\right)=\left(\left\{x_{1} / 1, x_{2} / 1, x_{3} / 1, x_{4} / 1\right\},\left\{y_{1} / 0, y_{2} / 0, y_{3} / 0, y_{4} / 0, y_{5} / 0\right\}\right)
\end{aligned}
$$

These $L$-fuzzy biconcepts do not provide information.

- Class $\mathcal{C}_{2}: E_{2}=\left\{x_{2}\right\}$

$$
\begin{aligned}
& \left(A_{2,1}, B_{2,1}\right)=\left(\left\{x_{1} / 0, x_{2} / 0.5, x_{3} / 0, x_{4} / 0\right\},\left\{y_{1} / 0.5, y_{2} / 1, y_{3} / 1, y_{4} / 0.5, y_{5} / 0.5\right\}\right) \\
& \left(A_{2,2}, B_{2,2}\right)=\left(\left\{x_{1} / 0, x_{2} / 1, x_{3} / 0, x_{4} / 0\right\},\left\{y_{1} / 0, y_{2} / 1, y_{3} / 1, y_{4} / 0, y_{5} / 0\right\}\right) \\
& \left(A_{2,3}, B_{2,3}\right)=\left(\left\{x_{1} / 0.5, x_{2} / 1, x_{3} / 0.5, x_{4} / 0.5\right\},\left\{y_{1} / 0, y_{2} / 0.5, y_{3} / 0.5, y_{4} / 0, y_{5} / 0\right\}\right)
\end{aligned}
$$


$x_{2}$ likes $y_{2}$ and $y_{3}$, and vice versa.

- Class $\mathcal{C}_{3}: E_{3}=\left\{x_{4}\right\}$

$$
\begin{aligned}
& \left(A_{3,1}, B_{3,1}\right)=\left(\left\{x_{1} / 0.5, x_{2} / 0.5, x_{3} / 0.5, x_{4} / 1\right\},\left\{y_{1} / 0.5, y_{2} / 0, y_{3} / 0, y_{4} / 0.5, y_{5} / 0.5\right\}\right) \\
& \left(A_{3,2}, B_{3,2}\right)=\left(\left\{x_{1} / 0, x_{2} / 0, x_{3} / 0, x_{4} / 0.5\right\},\left\{y_{1} / 1, y_{2} / 0.5, y_{3} / 0.5, y_{4} / 1, y_{5} / 1\right\}\right) \\
& \left(A_{3,3}, B_{3,3}\right)=\left(\left\{x_{1} / 0, x_{2} / 0, x_{3} / 0, x_{4} / 1\right\},\left\{y_{1} / 1, y_{2} / 0, y_{3} / 0, y_{4} / 0.5, y_{5} / 1\right\}\right)
\end{aligned}
$$

$x_{4}$ likes $y_{1}$ and $y_{5}$, and less $y_{4}$.

- Class $\mathcal{C}_{4}: E_{4}=\left\{x_{1}, x_{3}, x_{4}\right\}$

$$
\begin{aligned}
& \left(A_{4,1}, B_{4,1}\right)=\left(\left\{x_{1} / 0.5, x_{2} / 0, x_{3} / 0.5, x_{4} / 0.5\right\},\left\{y_{1} / 1, y_{2} / 0.5, y_{3} / 0.5, y_{4} / 1, y_{5} / 0.5\right\}\right) \\
& \left(A_{4,2}, B_{4,2}\right)=\left(\left\{x_{1} / 0.5, x_{2} / 0, x_{3} / 0.5, x_{4} / 1\right\},\left\{y_{1} / 1, y_{2} / 0, y_{3} / 0, y_{4} / 0.5, y_{5} / 0.5\right\}\right) \\
& \left(A_{4,3}, B_{4,3}\right)=\left(\left\{x_{1} / 1, x_{2} / 0.5, x_{3} / 1, x_{4} / 1\right\},\left\{y_{1} / 0.5, y_{2} / 0, y_{3} / 0, y_{4} / 0.5, y_{5} / 0\right\}\right)
\end{aligned}
$$

Everybody in $\left\{x_{1}, x_{3}, x_{4}\right\}$ likes $y_{1}$ and vice versa. And with a lower exigence level, also holds for $y_{4}$.

- Class $\mathcal{C}_{5}: E_{5}=\left\{x_{1}, x_{4}\right\}$

$$
\left(A_{5,1}, B_{5,1}\right)=\left(\left\{x_{1} / 1, x_{2} / 0, x_{3} / 0.5, x_{4} / 1\right\},\left\{y_{1} / 1, y_{2} / 0, y_{3} / 0, y_{4} / 0.5, y_{5} / 0\right\}\right)
$$

$x_{1}$ and $y_{1}$ like each other. The same information is obtained for $x_{4}$ and $y_{1}$. If we lower the demand level, a similar result holds for $y_{4}$.

This classification provides an useful tool to analyze the behavior of each one of the objects studying only those classes in which it appears.

In order to farther deepen the study, we want to set up similarity relations for the object and attribute sets. This similarity relations will provide us information about the relation among the different objects and attributes.

With this purpose, we will consider some results of L. Valverde [20], who defined an $F$-indistinguishability operator on a set $X$ as a reflexive, symmetric and $F$-transitive binary relation (being $F$ a continuous $t$-norm). He also proved that this operator can be generated by a family of fuzzy subsets of $X$.

If we consider representative elements of the equivalence classes, using the following theorem of L. Valverde [20], we can obtain similarity relations for the object set (analogously for the attribute set). These relations allow to classify, in a fuzzy way, the set of objects (or the set of attributes). 


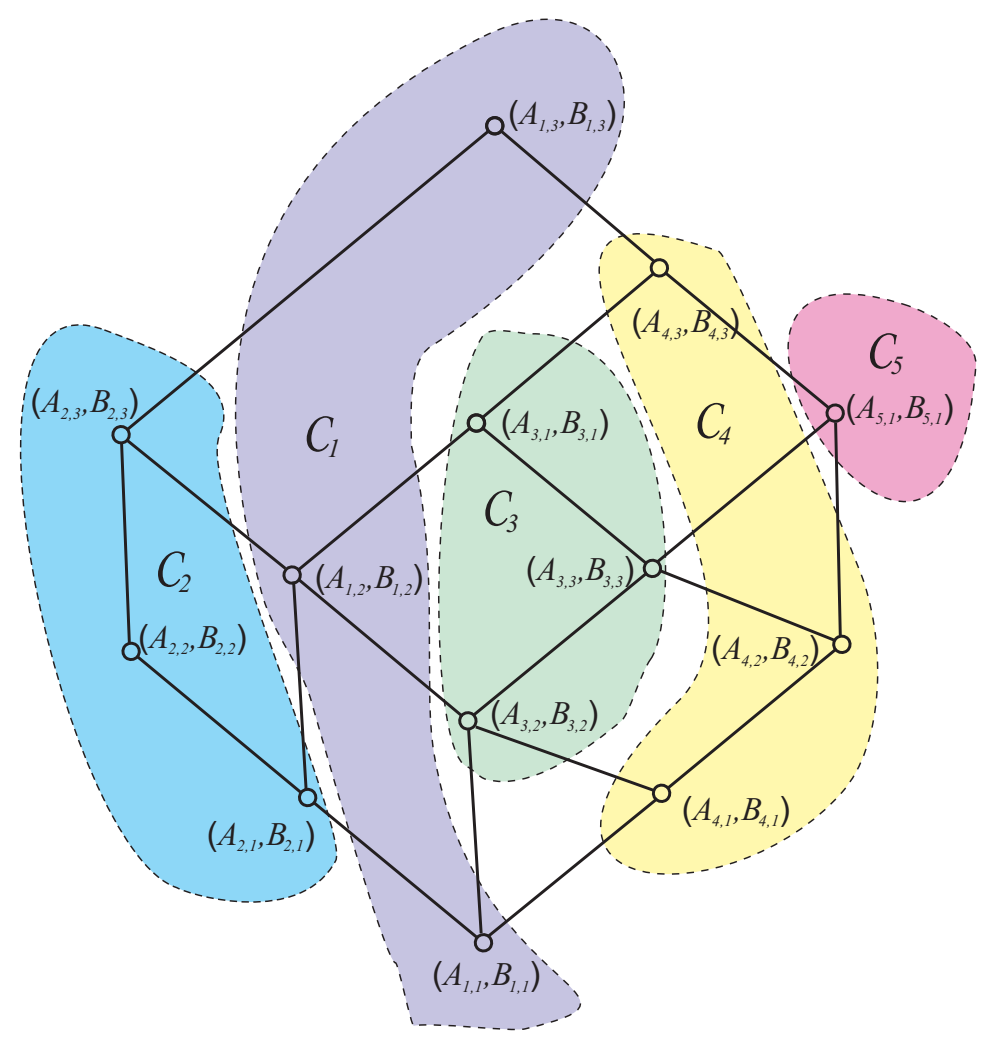

Figure 2: Classification of the $L$-fuzzy biconcepts.

Theorem 6. (Representation Theorem). Consider $S$ a map from $X \times X$ in $[0,1]$ and consider $F$ a continuous $t$-norm. Then, $S$ is a F-indistinguishability if and only if exists a family of fuzzy sets of $X,\left\{h_{j}\right\}, j \in J$ such that for every $x, y \in X$,

$$
S(x, y)=\inf _{j \in J} \vec{F}\left(\max \left(h_{j}(x), h_{j}(y)\right) \mid \min \left(h_{j}(x), h_{j}(y)\right)\right)
$$

where $\vec{F}(x \mid y)=\sup \{\alpha \in[0,1] \mid F(\alpha, x) \leq y\}$ is the residuation of the t-norm $F$.

If $F(x, y)=\operatorname{Min}(x, y)$, then the $F$-indistinguishability operator (similarity relation) can be expressed [20] in this way: 


$$
S(x, y)= \begin{cases}\inf _{j \in J_{x y}}\left(\min \left(h_{j}(x), h_{j}(y)\right)\right. & \text { if } J_{x y} \neq \emptyset \\ 1 & \text { otherwise }\end{cases}
$$

where $J_{x y}=\left\{j \in J / h_{j}(x) \neq h_{j}(y)\right\}$.

As representative element of each class we choose the extension of the supremum of its $L$-fuzzy concepts (or, respectively, the intension of the minimum of the $L$-fuzzy concepts of the class for the attributes). Since the $L$-fuzzy biconcepts gathered in a class share a common meaning, the choice of a different representative element may change the obtained similarity relation but does not provide a result contradicting the here proposed one.

Example 8. If we come back to our example, we can take the family $\left\{h_{j}\right\}, j \in$ $J$ of the concepts extensions representative of the different classes $\mathcal{C}_{j}$ (the supremum of each class):

$$
\begin{aligned}
h_{1} & =\left\{x_{1} / 1, x_{2} / 1, x_{3} / 1, x_{4} / 1\right\} \\
h_{2} & =\left\{x_{1} / 0.5, x_{2} / 1, x_{3} / 0.5, x_{4} / 0.5\right\} \\
h_{3} & =\left\{x_{1} / 0.5, x_{2} / 0.5, x_{3} / 0.5, x_{4} / 1\right\} \\
h_{4} & =\left\{x_{1} / 1, x_{2} / 0.5, x_{3} / 1, x_{4} / 1\right\} \\
h_{5} & =\left\{x_{1} / 1, x_{2} / 0, x_{3} / 0.5, x_{4} / 1\right\}
\end{aligned}
$$

and obtain the similarity relation $S$ (see Table 3 ).

Table 3: Similarity relation

\begin{tabular}{c|cccc}
$S$ & $x_{1}$ & $x_{2}$ & $x_{3}$ & $x_{4}$ \\
\hline$x_{1}$ & 1 & 0 & 0.5 & 0.5 \\
$x_{2}$ & 0 & 1 & 0 & 0 \\
$x_{3}$ & 0.5 & 0 & 1 & 0.5 \\
$x_{4}$ & 0.5 & 0 & 0.5 & 1
\end{tabular}

Since three different values appear in the similarity relation, hence we have three different $\alpha$-cuts $S_{1}, S_{0.5}$ and $S_{0}$ :

$$
S_{1}: x_{1} x_{2} x_{3} x_{4} \quad S_{0.5}: x_{1} x_{3} x_{4} x_{2} \quad S_{0}: x_{1} x_{2} x_{3} x_{4}
$$


For example, the $\alpha$-cut $S_{0.5}$ could be interpreted saying that the existing relationship between $x_{1}, x_{3}$ and $x_{4}$ is stronger than the one they have with $x_{2}$.

These similarity relations allow to establish fuzzy classifications in the object set.

This process could also be done from the point of view of the attributes, establishing different classes among them.

\section{Conclusions and future work}

The modeling of the use of two relations in an $L$-fuzzy context is the main goal of this work. We have defined the $L$-fuzzy bicontexts and study their associated $L$-fuzzy biconcepts.

Some interesting properties have been proved to set up a relationship between the $L$-fuzzy biconcepts and the $L$-fuzzy concepts obtained from the $L$-fuzzy contexts associated with each one of the relations. Moreover, in order to interpret the $L$-fuzzy biconcepts, we have defined a classification and similarity relations among the objects and attributes.

Finally, these results have been illustrated by means of an example about the relationship between two groups of people.

As future lines, we are interested in the study of the $L$-fuzzy bicontexts when $X=Y$. In the practical case, they can model the study of the opinion of a group of people about themselves or preference studies.

\section{Acknowledgments}

This work has been partially supported by the Research Group "Intelligent Systems and Energy (SI+E)" of the Basque Government, under Grant IT677-13, by the Research Groups "Artificial Intelligence and Approximate Reasoning" and "Adquisición de conocimiento y minería de datos, funciones especiales y métodos numéricos avanzados" of the Public University of Navarra and by project TIN2013-40765-P.

\section{References}

[1] C. Alcalde, A. Burusco, and R. Fuentes-González. Some results on the composition of l-fuzzy contexts. In Advances in Computational Intelligence, volume 298 of CCIS, pages 305-314. Springer, 2012. 
[2] C. Alcalde, A. Burusco, R. Fuentes-González, and I. Zubia. Treatment of $L$-fuzzy contexts with absent values. Information Sciences, 179(12):1-15, 2009 .

[3] R. Belohlavek. Fuzzy closure operators. Journal of Mathematical Analysis and Applications, 262:473-491, 2001.

[4] R. Belohlavek. Fuzzy relational Systems. Foundations and Principles. Kluwer Academic, 2002.

[5] R. Belohlavek and J. Konecny. Concept lattices of isotone vs. antitone Galois connections in graded setting: Mutual reducibility revisited. Information Sciences, 199:133-137, 2012.

[6] A. Burusco and R. Fuentes-González. Extracción de información relevante en contextos L-Fuzzy. In Proc. XI Congreso Español sobre Tecnologías y Lógica Fuzzy (ESTYLF 2002).

[7] A. Burusco and R. Fuentes-González. The study of the $L$-fuzzy concept lattice. Mathware and Soft Computing, I(3):209-218, 1994.

[8] A. Burusco and R. Fuentes-González. Concept lattices associated to W-contexts. Mathware and Soft Computing, 3(3):321-327, 1996.

[9] A. Burusco and R. Fuentes-González. Construction of the $L$-fuzzy concept lattice. Fuzzy Sets and Systems, 97(1):109-114, 1998.

[10] P. Cousot and R. Cousot. Constructive versions of Tarski's fixed point theorems. Pacific Journal of Mathematics, 82:43-57, 1979.

[11] Y. Djouadi and H. Prade. Interval-valued fuzzy galois connections: Algebraic requirements and concept lattice construction. Fundamenta Informaticae, 99(2):169-186, 2010.

[12] B. Ganter and R. Wille. Formal concept analysis: Mathematical foundations. Springer, Berlin - New York, 1999.

[13] L. J. Kohout and W. Bandler. Use of fuzzy relations in knowledge representation, acquisition and processing. In L. Zadeh and J. Kacprzyk, editors, Fuzzy logic for management of uncertainty, pages 415-435. 1992. 
[14] O. Krídlo, S. Krajči, and M. Ojeda-Aciego. The category of L-Chu correspondences and the structure of L-Bonds. Fundamenta Informaticae, 115:297-325, 2012.

[15] J. Medina. Multi-adjoint property-oriented concept lattices. Information Sciences, 190:95-106, 2012.

[16] J. Medina and M. Ojeda-Aciego. Multi-adjoint t-concept lattices. Information Sciences, 180(5):712-725, 2010.

[17] J. Medina and M. Ojeda-Aciego. Dual multi-adjoint concept lattices. Information Sciences, 225:47-54, 2013.

[18] S. Pollandt. Fuzzy Begriffe. Springer, 1997.

[19] A. Tarski. A lattice theoretical fixpoint theorem and its applications. Pacific Journal of Mathematics, 5:285-310, 1995.

[20] L. Valverde. On the structure of F-indistinguishability operators. Fuzzy Sets and Systems, 17:313-328, 1985.

[21] R. Wille. Restructuring lattice theory: An approach based on hierarchies of concepts. In Ivan Rival, editor, Ordered Sets, volume 83 of NATO Advanced Study Institutes Series, pages 445-470. Springer Netherlands, 1982. 